Welche(r) jüngere Ärztin/Arzt sieht seine Zukunft in einer gutgehenden

ganzheitsmedizinischen (speziell OM) Allgemeinpraxis

an einem besonders attraktiven Arbeitsort am Zürichsee?

Sie können bei Praxisübernahme von einem sehr guten Ruf profitieren und finden einen überdurchschnittlich gesundheitsbewussten Patientenstamm vor (ideal auch für seriöses Anti-Aging Konzept).

Melden Sie sich bitte schriftlich mit Ihrem Curriculum und evt. Vorstellungen unter:

Stichwort «OM-Praxis»

c/o S. Karger Verlag für Medizin und Naturwissenschaften GmbH・Wilhelmstrasse 20A 79098 Freiburg DEUTSCHLAND

Ihre Angaben werden selbstverständlich ganz vertraulich behandelt.

\title{
KARGER
}

Hier könnte Ihr Fort- und Weiterbildungsangebot stehen!

Nutzen Sie SCHWEIZERISCHE ZEITSCHRIFT FÜR

GANZHEITSMEDIZIN zur effektiven Kommunikation mit Ihrer Zielgruppe! So schaffen Sie die besten Voraussetzungen, sowohl gedruckt als auch online gesucht und gefunden zu werden.

Informationen und Angebote bei: Stefan Diekmann

Tel. +49 761 45207-13

s.diekmann@karger.de

\section{ASA TCM-Kongress 2010}

4. Fortbildungstage für Traditionelle Chinesische Medizin

$4^{\text {èmes }}$ Journées de Perfectionnement de Médecine Traditionnelle Chinoise

4. Giorni di Perfezionamento di Medicina Tradizionale Cinese

\section{Congress Center Basel}

9.-10. Dezember 2010

9-10 décembre 2010

Weitere Informationen

sowie Frühbucher-Rabatt siehe /

Ultérieures informations ainsi que pour

connaitre les avantages d'une

réservation précoce, veuillez consulter

\section{www.congress-info.ch/asa-tcm}

www.akupunktur-tcm.ch

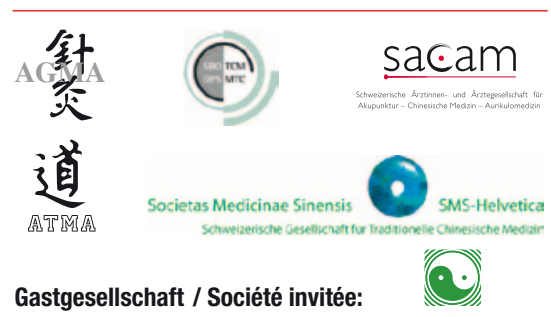

DÄGfA

\section{Der TCM-Kongress in der Schweiz! Le congrès de MTC en Suisse!}

\section{Top-Programm mit / Programme de qualité avec}

- 5 Hauptreferaten / 5 conférences principales avec traduction simultanée: - Andreas Noll, Berlin - Michael Hammes, Lerngo - Peter Firebrace, London

- Bernard de Wurstemberer, Vandoeuvres - Michael Wullinger, Rosenheim

- 14 Seminaren / 14 séminaires

- 4 Perlen / 4 perles

- 1 Journal Review

- Preisträger-Seminar / séminaire du lauréat

- Satelliten-Symposium / symposium satellite

\begin{tabular}{|l|l|l|}
\hline & 9.12 .2010 & 10.12 .2010 \\
\hline ASA & 8 Credits / Crédits & 7 Credits / Crédits \\
\hline SBO-TCM / OPS-MTC & 8 Credits / Crédits & 7 Credits / Crédits \\
\hline SGAM / SSMG & $\begin{array}{l}\text { Volle Fortbildung anrechenbar / } \\
\text { Durée entière reconnue }\end{array}$ \\
\hline SGGG / SSGO & $\begin{array}{l}8 \text { Credits allg. Fortbildung / } \\
8 \text { crédits perfectionnement général }\end{array}$ \\
\hline
\end{tabular}

